\title{
Search for Single-Top Production in ep Collisions at HERA
}

\section{S. Antonelli, L. Bellagamba and Katarzyna Wichmann* on behalf of the ZEUS Collaboration \\ DESY \\ E-mail: stefano.antonelli@cnaf.infn.it}

\begin{abstract}
Results of a recent search for single-top production in $e^{ \pm} p$ collisions at HERA are presented. The search for single-top production, $e p \rightarrow e t X$, has been performed with the ZEUS detector at HERA collider using data corresponding to an integrated luminosity of $0.37 \mathrm{fb}^{-1}$. No evidence for top production was found, consistent with the expectation from the Standard Model. Limits were computed for single-top production via flavour changing neutral current transitions. The result was combined with a previous ZEUS result yielding a total luminosity of $0.50 \mathrm{fb}^{-1}$. A $95 \%$ Credibility Level upper limit of $0.13 \mathrm{pb}$ was obtained for the cross section at the centre-of-mass energy of $\sqrt{s}=315 \mathrm{GeV}$.
\end{abstract}

36th International Conference on High Energy Physics,

July 4-11, 2012

Melbourne, Australia

${ }^{*}$ Speaker. 


\section{Introduction}

In $e p^{1}$ collisions at HERA, the production of single-top quark is possible due to the large centre-of-mass energy $\sqrt{s}=318 \mathrm{GeV}$. The dominant production process of single top quarks in the Standard Model (SM) is the charged current (CC) deep inelastic scattering (DIS) reaction $e p \rightarrow v t X[1,2]$ which has a cross section of less than $1 \mathrm{fb}$.

No sizeable production is hence expected in our data sample and any excess can be attributed to new physics. In several extensions of the SM [3], single-top production can happen via a flavour changing neutral current (FCNC) process mediated by an effective coupling which allows a $u-t$ or $c-t$ transition via a neutral vector boson $(\gamma$ or $Z$ ), see Fig.1.

Owing to the large $Z$ mass, this process is more sensitive to a coupling of the type $t q \gamma$. Furthermore, large values of $x$, the fraction of the proton momentum carried by the struck quark, are needed to produce a top quark. Since the $u$-quark parton distribution function (PDF) of the proton is dominant at large $x$, the production of single top quark is most sensitive to the tu $\gamma$ coupling.

The analysis has been performed with $0.37 \mathrm{fb}^{-1}$ and extends the previously pub-

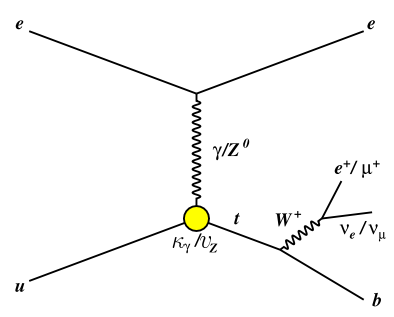

Figure 1: Anomalous single-top production via flavour changing neutral current transitions at HERA with subsequent decays $t \rightarrow b W^{+}$and $W^{+} \rightarrow$ $v_{e}\left(v_{\mu}\right) e^{+}(\mu+)$. lished ZEUS results [4] corresponding to $0.13 \mathrm{fb}^{-1}$. Limits for single-top production via FCNC were computed combining this result with the previous ZEUS one [4] for a total luminosity of $0.50 \mathrm{fb}^{-1}$.

\section{Event selection}

The event selection was optimised for single-top production via photon exchange, looking for the dominant decay $t \rightarrow b W$ and subsequent $W$ decay to $e$ and $\mu$ and their respective neutrinos. The selection is based on requiring an isolated high- $p_{T}$ lepton, large missing transverse momentum and high hadronic $P_{T}$.

The main preselection cuts were the following:

- $P_{T, \text { miss }}>10(12) \mathrm{GeV} \mu$ - (e-) channel;

- leptonic $p_{T}>8$ (10) $\mathrm{GeV} \mu$ - (e-) channel;

- transverse mass $M_{T}>10 \mathrm{GeV}$ e-channel only;

The main final cuts where the following:

- hadronic $P_{T}>40 \mathrm{GeV}$ for both channels;

- $P_{T, \text { miss }}>15 \mathrm{GeV}$ e-channel.

\footnotetext{
${ }^{1}$ Here and in the following, $e$ denotes both the electron and the positron.
} 
Figure 2 shows the preselection plots in the muon (left) and electron (right) channels. Black dots are data, green area is $\mathrm{MC}$ and the dark-shaded region is the $W$ contribution; reasonable agreement is observed in all cases.

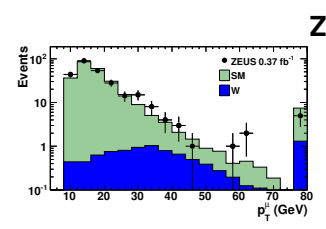

ZEUS
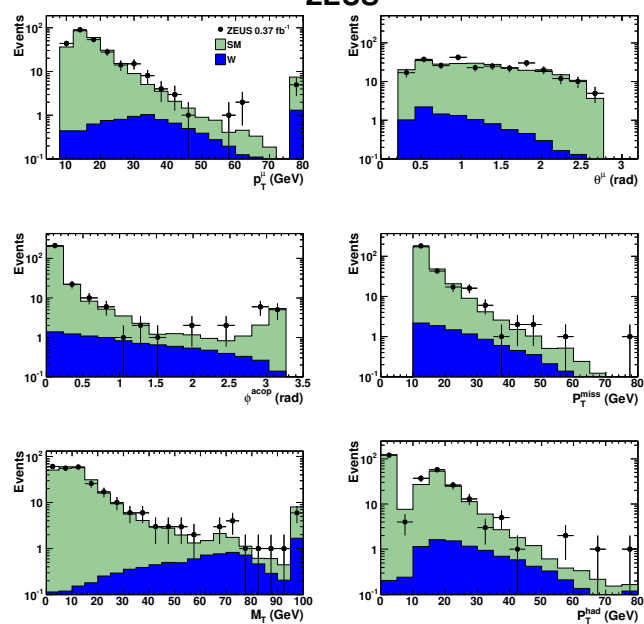
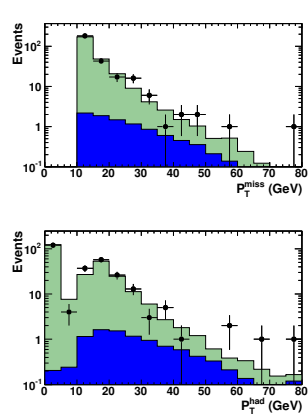

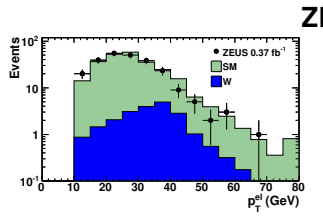

ZEUS
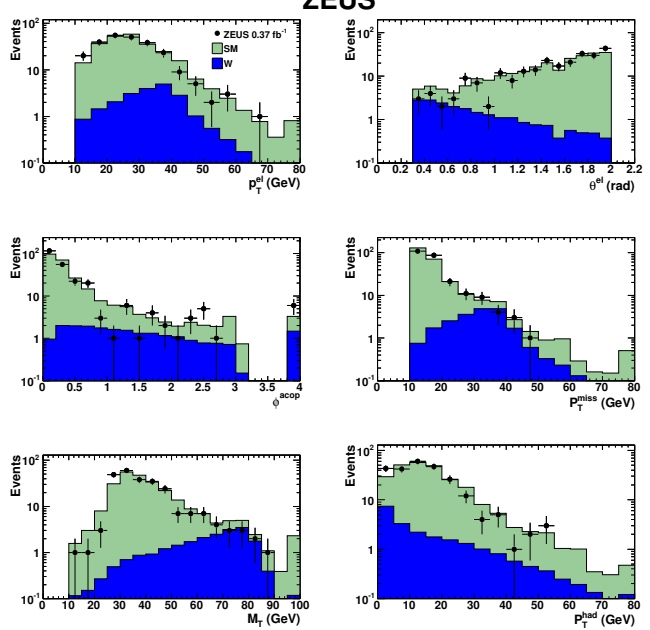

Figure 2: Preselection plots in the muon (left) and electron (right) channels. Black dots are data, green area is MC and the dark-shaded region is the $W$ contribution.

\section{Systematic uncertainties}

The main contribution to the systematical uncertainties on the predicted SM events is due to the following sources:

- the theoretical uncertainty on the $\mathrm{W}$ background normalisation; $\pm 15 \%$;

- the statistical uncertainty on the total SM prediction after the final selection; $\pm 13 \%$ and $\pm 9 \%$ for the $e$ - and $\mu$-channel respectively;

- the uncertainty on the NC DIS background; $\pm 15 \%$ for the preselection and $\pm 6 \%$ for the final selection in the $e$-channel and negligible in the $\mu$-channel.

\section{Limits evaluation}

Since no excess of events above the SM expectations is observed, a further selection is made to evaluate the limit on FCNC cross section under the assumption of no signals. The 95\% Credibility Level (C.L.) limit on the cross section is found to be: $\sigma<0.24 \mathrm{pb}$ at $\sqrt{s}=318 \mathrm{GeV}$. The limit on the cross section is converted into a limit on the coupling $\kappa_{\gamma}<0.18$ (95\% C.L.). This limit has been combined with a previous ZEUS result [4] giving the following constraints: $\sigma<0.13 \mathrm{pb}$ and $\kappa_{\gamma}<0.13$ (95\% C.L.) [5]. Constraints on the anomalous top branching ratios $t \rightarrow u \gamma\left(\mathrm{Br}_{\mathrm{u} \gamma}\right)$ and $t \rightarrow u Z\left(\mathrm{Br}_{\mathrm{uZ}}\right)$ were also evaluated assuming a non-zero $v_{Z}$. Figure 3 shows the ZEUS boundary in the $\left(\mathrm{Br}_{\mathrm{u} \gamma}, \mathrm{Br}_{\mathrm{uZ}}\right)$ plane compared to limits from H1 [6], ALEPH [7], CDF [8], D0 [9]. For low values of $v_{Z}$, resulting in branching ratios of $t \rightarrow u Z$ of less than $4 \%$, this paper provides the current best limits. 


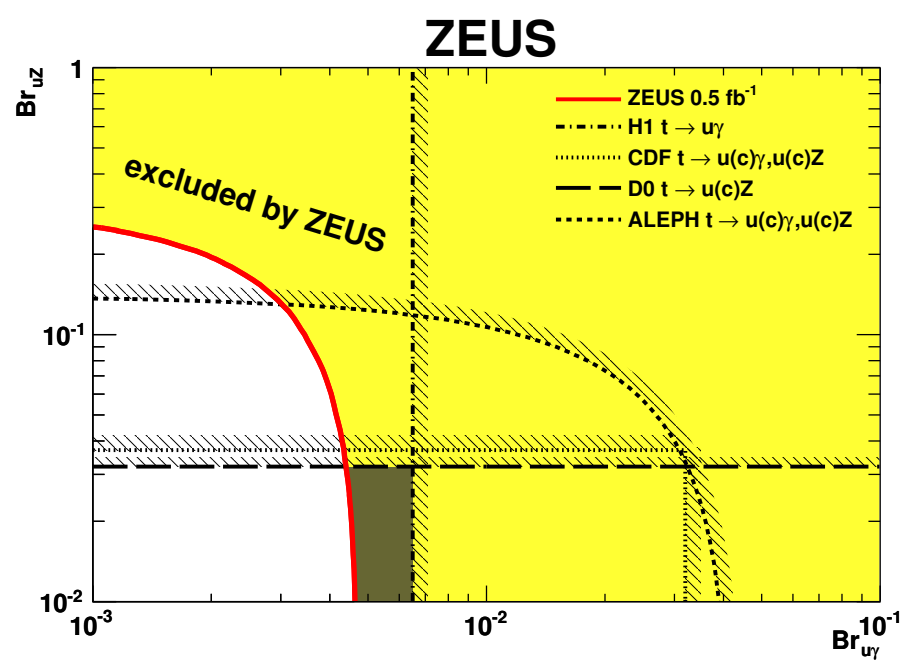

Figure 3: ZEUS boundary in the $\left(\mathrm{Br}_{\mathrm{u} \gamma}, \mathrm{Br}_{\mathrm{uz}}\right)$ plane. Also shown are boundaries of $\mathrm{H1}$ [6], CDF [8], D0 [9] and ALEPH [7]. The shaded area is excluded. The dark shaded region denotes the area uniquely excluded by ZEUS.

\section{Conclusions}

A search for possible deviations from the Standard Model predictions due to flavour- changing neutral current top production in events with high- $p_{T}$ leptons and high missing transverse momentum was performed using an integrated luminosity of $0.37 \mathrm{fb}^{-1}$, collected by the ZEUS detector in 2004-2007. Since no significant deviation from the expectation was observed, the results were used to set limits on the anomalous production of single top at HERA. A 95\% C.L. upper limit on the cross section of $\sigma<0.24 \mathrm{pb}$ at a centre-of-mass energy of $318 \mathrm{GeV}$ was obtained. The limit was combined with a previous ZEUS result [4], obtained using HERA I data, for a total integrated luminosity of $0.50 \mathrm{fb}^{-1}$, giving a combined $95 \%$ credibility-level upper limit of $\sigma<0.13 \mathrm{pb}$ at $\sqrt{s}=315 \mathrm{GeV}$. This limit, assuming a vanishing coupling of the top quark to the $Z$ boson $\left(v_{Z}\right)$, corresponds to a constraint on the coupling of the top to the $\gamma$ of $\kappa_{\gamma}<0.13$. Constraints on the anomalous top branching ratios $t \rightarrow u \gamma$ and $t \rightarrow u Z$ were also evaluated assuming a non-zero $v_{Z}$. For low values of $v_{Z}$, resulting in branching ratios of $t \rightarrow u Z$ of less than $4 \%$, see Fig. 3, this paper provides the current best limits.

\section{References}

[1] T. Stelzer, D. Sullivan and S. Willenbrock, Phys. Rev. D56 (1997) 5919.

[2] T. Moretti and K. Odagiri, Phys. Rev. D57 (1998) 3040.

[3] T.Han, J.L.Hewett, Phys. Rev. D60 (1999) 074015.

[4] ZEUS Coll., S. Chekanov et al., Phys. Lett. B559 (2003) 153

[5] ZEUS Coll., H. Abramowicz et al., Phys. Lett. B708 (2012) 27

[6] H1 Coll., F.D. Aaron et al., Phys. Lett. B678 (2009) 450 
[7] Aleph Coll., A. Heister et al., Phys. Lett. B543 (2002) 173

[8] CDF Coll., F. Abe et al., Phys. Rev. Lett. 80 (1998) 2525

[9] D0 Coll., V.M. Abazov et al., Phys. Lett. B701 (2011) 313 